\title{
Stable many-to-many matchings with contracts
}

Citation for published version (APA):

Klaus, B. E., \& Walzl, M. (2006). Stable many-to-many matchings with contracts. METEOR, Maastricht University School of Business and Economics. METEOR Research Memorandum No. 042 https://doi.org/10.26481/umamet.2006042

Document status and date:

Published: 01/09/2006

DOI:

10.26481/umamet.2006042

Document Version:

Publisher's PDF, also known as Version of record

\section{Please check the document version of this publication:}

- A submitted manuscript is the version of the article upon submission and before peer-review. There can be important differences between the submitted version and the official published version of record.

People interested in the research are advised to contact the author for the final version of the publication, or visit the DOI to the publisher's website.

- The final author version and the galley proof are versions of the publication after peer review.

- The final published version features the final layout of the paper including the volume, issue and page numbers.

Link to publication

\footnotetext{
General rights rights.

- You may freely distribute the URL identifying the publication in the public portal. please follow below link for the End User Agreement:

www.umlib.nl/taverne-license

Take down policy

If you believe that this document breaches copyright please contact us at:

repository@maastrichtuniversity.nl

providing details and we will investigate your claim.
}

Copyright and moral rights for the publications made accessible in the public portal are retained by the authors and/or other copyright owners and it is a condition of accessing publications that users recognise and abide by the legal requirements associated with these

- Users may download and print one copy of any publication from the public portal for the purpose of private study or research.

- You may not further distribute the material or use it for any profit-making activity or commercial gain

If the publication is distributed under the terms of Article $25 \mathrm{fa}$ of the Dutch Copyright Act, indicated by the "Taverne" license above, 


\section{Bettina Klaus, Markus Walzl}

Stable Many-to-Many Matchings with Contracts

$\mathrm{RM} / 06 / 042$

JEL code: C62, C78, D78, J41

\section{METE@R}

Maastricht research school of Economics of TEchnology and ORganizations

Universiteit Maastricht

Faculty of Economics and Business Administration P.O. Box 616

NL - 6200 MD Maastricht

phone : ++31433883830

fax $\quad$ : ++31433884873 



\title{
Stable Many-to-Many Matchings with Contracts
}

\author{
Bettina Klaus* $\quad$ Markus Walzl ${ }^{\dagger}$
}

September 2006

\begin{abstract}
We consider several notions of setwise stability for many-to-many matching markets with contracts and provide an analysis of the relations between the resulting stable sets and pairwise stable sets for general, substitutable, and strongly substitutable preferences. Apart from obtaining "set inclusion results" on all three domains, we prove that for substitutable preferences the set of pairwise stable matchings is nonempty and coincides with the set of weakly setwise stable matchings. For strongly substitutable preferences the set of pairwise stable matchings coincides with the set of setwise stable matchings. We also show that Roth's (1984b) stability coincides with pairwise stability for substitutable preferences.
\end{abstract}

JEL classification: C62, C78, D78, J41.

Keywords: Many-to-Many Matching, Matching with Contracts, Pairwise Stability, Setwise Stability.

${ }^{*}$ Corresponding author: Department of Economics, Maastricht University, P.O. Box 616, 6200 MD Maastricht, The Netherlands; e-mail: B.Klaus@algec.unimaas.nl

${ }^{\dagger}$ Department of Economics, Maastricht University, P.O. Box 616, 6200 MD Maastricht, The Netherlands; e-mail: M.Walzl@algec.unimaas.nl 


\section{Introduction}

We consider a general class of two-sided many-to-many matching markets, so-called matching with contracts markets (Roth, 1984b). ${ }^{1}$ The matching with contracts model contains classical two-sided matching markets such as marriage and college admissions markets (e.g., Gale and Shapley, 1962), job matching markets (e.g., Roth, 1985; Kelso and Crawford, 1982), ${ }^{2}$ and certain auction markets (e.g., Milgrom, 2004). ${ }^{3}$ Throughout the article, without loss of generality, we model matching markets with contracts as trading platforms where buyers and sellers interact: there is a set of bilateral contracts between buyers and sellers that specify the trading conditions (e.g., the set of items that are bought/sold, delivery dates, prices, service agreements, etc.). Moreover, buyers as well as sellers can trade with more than one agent on the other side of the market at the same time. The agents' strict preferences over (feasible, legal, etc.) sets of contracts or allocations completes the description of a many-to-many matching market with contracts.

Stability is the central solution requirement derived from empirical as well as theoretical studies (e.g., Roth, 1982, 1984a, 1991; Roth and Xing, 1994). Loosely speaking, an allocation of contracts is "stable" if it is individually rational [no buyer or seller would prefer to cancel some of her contracts] and satisfies a no blocking requirement. Clearly, there are various ways for a set of agents to block a given allocation of contracts:

- pairwise blocking: a buyer and a seller would like to add a new joint contract or replace a previous joint contract while possibly canceling other contracts;

- setwise blocking: a set of buyers and sellers would like to implement a new set of contracts among themselves while possibly canceling other contracts.

In the case of setwise blocking, the blocking requirement can be refined by imposing extra conditions on when a set of agents is allowed to block a given allocation of contracts:

- strongly setwise blocking describes the minimal requirement for any setwise blocking (it is the benchmark without extra conditions): the agents in the blocking coalition should be better off with the new sets of contracts they receive through blocking;

- setwise blocking: the set of new contracts is a strong setwise block and individually rational;

- weakly setwise blocking: the set of new contracts is a setwise block, but blocking can only occur if agents in the blocking coalition receive their best set of contracts from the new and the previous contracts.

\footnotetext{
${ }^{1}$ Closely related markets are many-to-one matching markets with contracts (e.g., Hatfield and Milgrom, 2005) and many-to-many matching markets (e.g., Echenique and Oviedo, 2006; Konishi and Ünver, 2006; Sotomoyor, 1999).

${ }^{2}$ Roth and Sotomayor (1990) give a comprehensive and complete survey of these and related two-sided matching models up to 1990 .

${ }^{3}$ For a more detailed discussion see Hatfield and Milgrom (2005) or Haake and Klaus (2005).
} 
Clearly, the various setwise blocking concepts differ with respect to the admissibility of a blocking coalition. While strong setwise blocks only require an improvement upon the status quo, setwise blockings also require that the blocking allocation is individually rational (i.e., that none of the members of the blocking coalition has an incentive to cancel contracts unilaterally). Finally, weak setwise blocks require that there is no conflict in the blocking coalition (i.e., all members of the blocking coalition obtain their best set of contracts among their original contracts and the new contracts of the blocking coalition.). Weak setwisestability is a new stability concept that, as we will demonstrate in the paper, bridges the gap between pairwise stability and previously considered setwise stability notions.

For many matching models various of the blocking notions coincide (see for instance Hatfield and Milgrom's, 2005, many-to-one matching markets with contracts). Sotomoyor (1999) demonstrates that for many-to-many matching markets notions of pairwise and setwise stability indeed differ.

As Echenique and Oviedo (2006, page 233) point out, "Many-to-many matching markets are understood less well than many-to-one markets ...". In our opinion, one of the reasons is that different stability notions are used in various papers while their relationships are not well understood. After a description of the model, we therefore first introduce pairwise stability and the various notions of setwise stability as implied by the blocking requirements listed before. Then, the set of pairwise (weakly setwise, etc.) stable allocations equals the set of all allocations that cannot be pairwise (weakly setwise, etc.) blocked.

We then analyze the relations between the resulting stable sets for various standard preference domains and obtain the following set inclusion results:

- general preferences: strongly setwise stable allocations $\subseteq$ setwise stable allocations $\subseteq$ weakly setwise stable allocations $\subseteq$ pairwise stable allocations;

- substitutable preferences: strongly setwise stable allocations $\subseteq$ setwise stable allocations $\subseteq$ weakly setwise stable allocations $=$ pairwise stable allocations;

- strongly substitutable preferences: strongly setwise stable allocations $\subseteq$ setwise stable allocations $=$ weakly setwise stable allocations $=$ pairwise stable allocations .

Apart from obtaining set inclusion results on all three domains, we prove that for substitutable preferences the set of pairwise stable matchings is nonempty and coincides with the set of weakly setwise stable matchings. ${ }^{4}$ Hence weakly setwise stable matchings do exist for substitutable preferences, and weak setwise stability is indeed a setwise stability concept in between the familiar pairwise stability and previously considered setwise stability notions.

For strongly substitutable preferences the set of pairwise stable matchings coincides with the set of setwise stable matchings. Finally, we demonstrate that all set inclusion results are tight (Examples 1, 4, and 5). Note that some examples were known for many-to-many matching markets, but that by using the additional degree of freedom of different contracts between the same buyer and seller, we are able to construct examples with fewer agents.

\footnotetext{
${ }^{4}$ Furthermore, for substitutable preferences, (setwise) stability as introduced in Roth (1984b) coincides with pairwise stability as demonstrated in the Appendix.
} 


\section{Many-to-Many Matching Markets with Contracts}

\subsection{Buyers, Sellers, and Contracts}

We consider a model, in which buyers and sellers are matched to each other (alternatively, we could model many-to-many matching markets with contracts as job-matching markets or matching markets where clients are assigned to consultancy firms). Let $B$ denote the finite set of buyers, $S$ the finite set of sellers, and $N=B \cup S$ the set of agents. By $b$ we denote a generic buyer, by $s$ a generic seller, and by $i, j$ generic agents.

We model the typical features of a many-to-many matching market with contracts by assuming that each buyer can buy from several sellers and each seller can sell to several buyers. A (bilateral) contract specifies a trade between one buyer and one seller and further terms of trade such as, for instance, the set and quantity of items sold, the price, postal and handling fees, delivery time, guarantees for the product and delivery, and service agreements. Formally the set of contracts is described by a set $X$ in connection with a mapping $\mu=\left(\mu_{B}, \mu_{S}\right): X \rightarrow$ $B \times S$ that specifies the bilateral structure of each contract. So, for any contract $x \in X$, $\mu(x)=(b, s)$ means that contract $x$ is established between buyer $b$ and seller $s$. Note that for two contracts $x, x^{\prime} \in X, x \neq x^{\prime}$, with $\mu(x)=\mu\left(x^{\prime}\right), x$ and $x^{\prime}$ specify different contract terms between the same buyer and seller. By $X_{i}=\{x \in X \mid \mu(x)=(i, s)$ or $\mu(x)=(b, i)\}$ we denote the set of contracts that involve agent $i$.

If all sellers offer the same set of contract specifications $K$ to all buyers, then the set of contracts $X$ can be represented as a Cartesian product $X=B \times S \times K$. An example of such a contract specification $K$ would be a price scale that all sellers of a standardized product employ. However, note that sellers may not necessarily use the same contract specification: sellers may sell different types and numbers of products and even if they sell the same standardized products, a seller who is further away may have to charge higher shipment costs than a seller who is located closer to the buyer. For each agent it is always possible to reject any set of contracts, that is to not buy or sell certain items. We refer to the specific situation in which an agent does not buy or sell any item as a null contract, denoted by $\emptyset$.

As a special case consider pure matching contracts, i.e., any contract only consists of a match between a buyer and a seller such that $X=\{(b, s) \in B \times S\}$. This restricts our model to many-to-many matching markets as analyzed in Sotomoyor (1999), Echenique and Oviedo (2006), and Konishi and Ünver (2006).

\subsection{Buyers' and Sellers' Preferences}

No buyer $b$ is allowed to have more than one contract with a certain seller at the same time (buying several items from one seller is summarized in a single contract). Therefore, we define the set of feasible contracts for buyer $b$ by $\mathcal{X}_{b}:=\left\{X^{\prime} \subseteq X_{b} \mid\right.$ for all $\left.s \in S,\left|X^{\prime} \cap X_{s}\right| \leq 1\right\}$. Symmetrically to the buyers, no seller $s$ is allowed to have more than one contract with a buyer at the same time (selling several items to the same buyer is summarized in a single contract). The set of feasible contracts for seller $s$ is $\mathcal{X}_{s}:=\left\{X^{\prime} \subseteq X_{s} \mid\right.$ for all $\left.b \in B,\left|X^{\prime} \cap X_{b}\right| \leq 1\right\}$.

Note that for each agent $i$ the null contract is always feasible, i.e., $\emptyset \in \mathcal{X}_{i}$, and that $X^{\prime} \in \mathcal{X}_{i}$ implies for all $Y^{\prime} \subseteq X^{\prime}, Y^{\prime} \in \mathcal{X}_{i}$. Each agent $i$ has a total (linear) order over sets of feasible contracts $\mathcal{X}_{i}$ represented by a preference relation $R_{i}{ }^{5}$ Given $X^{\prime}, Y^{\prime} \in \mathcal{X}_{i}, X^{\prime} P_{i} Y^{\prime}$

\footnotetext{
${ }^{5}$ In other words, $R_{i}$ represents a binary relation that satisfies antisymmetry (for all $X^{\prime}, Y^{\prime} \in \mathcal{X}_{i}$, if $X^{\prime} R_{i} Y^{\prime}$
} 
means that agent $i$ strictly prefers the set of contracts $X^{\prime}$ to the set of contacts $Y^{\prime} ; X^{\prime} R_{i} Y^{\prime}$ means that $X^{\prime} P_{i} Y^{\prime}$ or $X^{\prime}=Y^{\prime}$ and that agent $i$ weakly prefers the set of contracts $X^{\prime}$ to the set of contacts $Y^{\prime}$. We denote the set of all possible total orders for agent $i$ by $\mathcal{R}_{i}$. Since preference relation $R_{i} \in \mathcal{R}_{i}$ is a total order, it induces a well-defined choice correspondence $C_{i}: 2^{X} \rightarrow \mathcal{X}_{i}$ that assigns to each set of contracts $X^{\prime} \subseteq X$ agent $i$ 's most preferred feasible set of contracts available for her in $X^{\prime} \cup\{\emptyset\}$, i.e., for all $X^{\prime} \subseteq X, C_{i}\left(X^{\prime}\right) \in \mathcal{X}_{i}, C_{i}\left(X^{\prime}\right) \subseteq X^{\prime} \cup\{\emptyset\}$, and there is no $Y \subseteq X^{\prime}, Y \in \mathcal{X}_{i}$, with $Y P_{i} C_{i}\left(X^{\prime}\right)$.

After having introduced the domain of general preferences, we will introduce two domains that have played an important role in two-sided matching, namely substitutability and strong substitutability: see for instance Echenique and Oviedo (2006) who also use these domains to analyze the relation between various solution concepts such as (setwise) stable sets and core(-like) solutions.

\subsection{Substitutability}

Loosely speaking, an agent has substitutable preferences if she does not consider complementarities in the sets of contracts. To be precise, the condition for substitutable preference states that if a contract is chosen by an agent from some set of contracts, then that contract is still chosen by the agent from a smaller set of contracts that include it. Formally, agent $i$ 's preferences $R_{i}$ are substitutable if

(SUB) for all sets of contracts $X^{\prime} \subseteq Y^{\prime} \subseteq X: X^{\prime} \cap C_{i}\left(Y^{\prime}\right) \subseteq C_{i}\left(X^{\prime}\right)$.

Equivalently one can formulate substitutability as follows (Hatfield and Milgrom, 2005). If a contract is not chosen by an agent from some set of contracts, then that contract is still not chosen by the agent from a larger set of contracts. For any set of contracts $X^{\prime} \subseteq X$, $N C_{i}\left(X^{\prime}\right):=X^{\prime} \backslash C_{i}\left(X^{\prime}\right)$ denotes the set of all contracts that are not chosen from set $X^{\prime}$ by choice correspondence $C_{i}$. One can easily prove that condition (SUB) is equivalent to the following condition (SUB').

(SUB') For all sets of contracts $X^{\prime} \subseteq Y^{\prime} \subseteq X: N C_{i}\left(X^{\prime}\right) \subseteq N C_{i}\left(Y^{\prime}\right)$.

\subsection{Strong Substitutability}

Essentially, the strong substitutable preference condition (Echenique and Oviedo, 2006) states that if a contract is chosen by an agent from some set of contracts, then that contract is still chosen by the agent from a worse set of contracts that include it. We first have to extend Echenique and Oviedo's definition of strong substitutability for many-to-many matching markets to many-to-many matching markets with contracts.

Let $i$ be an agent who participates in a many-to-many matching market. Recall that in such a "pure" matching market exactly one contract between any buyer and any seller exists. Hence, all subsets of $X_{i}$ are automatically feasible and one can easily extend agent $i$ 's preferences to all subsets of $X$ : for $X^{\prime}, Y^{\prime} \subseteq X,\left(X^{\prime} \cap X_{i}\right) P_{i}\left(Y^{\prime} \cap X_{i}\right)$ implies $X^{\prime} P_{i} Y^{\prime}$.

When allowing for different contracts between buyers and sellers, not all subsets of $X_{i}$ are feasible anymore and the agent's strict preferences over feasible contract sets cannot be straightforwardly extended to strict preferences over all subsets of $X_{i}$. Thus, while Echenique and $Y^{\prime} R_{i} X^{\prime}$, then $X^{\prime}=Y^{\prime}$ ), transitivity (for all $X^{\prime}, Y^{\prime}, Z^{\prime} \in \mathcal{X}_{i}$, if $X^{\prime} R_{i} Y^{\prime}$ and $Y^{\prime} R_{i} Z^{\prime}$, then $X^{\prime} R_{i} Z^{\prime}$ ), and comparability (for all $X^{\prime}, Y^{\prime} \in \mathcal{X}_{i}, X^{\prime} R_{i} Y^{\prime}$ or $Y^{\prime} R_{i} X^{\prime}$ ). 
and Oviedo (2006) can simply use the strict preference relation of an agent to compare two sets of contracts $X^{\prime}$ and $Y^{\prime}$, we have to use the agent's choice function to avoid having to extend preferences to compare sets of contracts that are not feasible. ${ }^{6}$

Formally, agent $i$ 's preferences $R_{i}$ are strongly substitutable if

(SSUB) for all sets of contracts $X^{\prime}, Y^{\prime} \subseteq X$ such that $C_{i}\left(Y^{\prime}\right) P_{i} C_{i}\left(X^{\prime}\right): X^{\prime} \cap C_{i}\left(Y^{\prime}\right) \subseteq C_{i}\left(X^{\prime}\right)$.

For many-to-many matching markets, our definition coincides with that of Echenique and Oviedo (2006). Equivalently one can formulate strong substitutability as follows. If a contract is not chosen by an agent from some set of contracts, then that contract is still not chosen by the agent from a set of contracts she considers better according to her choice function. One can easily prove that condition (SSUB) is equivalent to the following condition (SSUB').

(SSUB') For all sets of contracts $X^{\prime}, Y^{\prime} \subseteq X$ such that $C_{i}\left(Y^{\prime}\right) P_{i} C_{i}\left(X^{\prime}\right): N C_{i}\left(X^{\prime}\right) \cap Y^{\prime} \subseteq$ $N C_{i}\left(Y^{\prime}\right)$.

It is clear that if preferences are strongly substitutable, then they are also substitutable. For a discussion of strong substitutability in comparison to other classical matching domain restrictions (e.g., separability and/or responsiveness) see Echenique and Oviedo (2006, Section 6.3).

\subsection{Allocations in Many-to-Many Matching Markets with Contracts}

Since the set of contracts $X$ and the set of agents $N$ remain fixed throughout this study, we denote a (many-to-many) matching market (with contracts) by a preference profile $R=$ $\left(R_{i}\right)_{i \in N}$. The set of all preference profiles is denoted by $\mathcal{R}$.

For any set of contracts $A \subseteq X$ and any agent $i$ we denote by $A_{i}=A \cap X_{i}$ all contracts in $A$ that involve agent $i$. An allocation is a set of contracts $A \subseteq X$ such that (i) for any buyer $b,\left[A_{b} \in \mathcal{X}_{b}\right]$ and $\left[x \in A_{b} \cap X_{s}\right.$ implies $\left.x \in A_{s}\right]$ and (ii) for any seller $s,\left[A_{s} \in \mathcal{X}_{s}\right]$ and $\left[x \in A_{s} \cap X_{b}\right.$ implies $\left.x \in A_{b}\right]$.

We denote the set of allocations by $\mathcal{A}$. Clearly, all preference relations $R_{i}$ induce weak preferences over allocations in a natural way. We use the same notation for preferences over feasible contract sets and allocations: for all agents $i \in N$ and allocations $A, A^{\prime} \in \mathcal{A}, A R_{i} A^{\prime}$ if and only if $A_{i} R_{i} A_{i}^{\prime}$.

\section{Pairwise and Setwise Stability}

As described in the Introduction, an important criterion for an allocation to be accepted as final outcome is stability. Next, we introduce various notions of stability.

\subsection{Individual Rationality and Pairwise Stability}

First, since the matching markets we consider here are based on voluntary participation, a necessary condition for allocation $A$ to be stable is individual rationality:

At any allocation $A$ each agent $i$ who is assigned a set of contracts $A_{i} \neq \emptyset$ can reject some or all contracts in $A_{i}$. Thus, an allocation $A$ is individually rational for matching market $R \in \mathcal{R}$ if for all $i \in N$ and $X^{\prime} \subseteq A_{i}, A_{i} R_{i} X^{\prime}$. Alternatively, $A$ is individually rational if

\footnotetext{
${ }^{6}$ We could also use the following preference extension: for $X^{\prime}, Y^{\prime} \subseteq X, C_{i}\left(X^{\prime}\right) R_{i} C_{i}\left(Y^{\prime}\right)$ implies $X^{\prime} R_{i} Y^{\prime}$.
} 
(IR) for all $i \in N, C_{i}(A)=A_{i}$

By $I R(R) \subseteq \mathcal{A}$ we denote the set of individually rational allocations for matching market $R$.

The notion of individual rationality we use here is the same as in Echenique and Oviedo (2006), Hatfield and Milgrom (2005), and Roth (1984b). Konishi and Ünver (2006) refer to individual rationality as "individual stability."

We continue with a weak notion of stability - pairwise stability - that plays a central role in all previous articles on (many-to-many) matching (e.g., Roth, 1984b; Echenique and Oviedo, 2006).

Let $b \in B$ and $s \in S$, and consider an individually rational allocation $A$. We define pairwise blocking of an allocation $A$ by buyer $b$ and seller $s$ as follows.

Assume that no $y \in A$ such that $\mu(y)=(b, s)$ exists (buyer $b$ and seller $s$ do not have a contract at $A$ ). Then, $b$ and $s$ can block allocation $A$ if there is a contract $x \in X, \mu(x)=(b, s)$, such that $b$ and $s$ strictly prefer adding $x$ to their respective sets of contracts $A_{b}$ and $A_{s}$ while possibly canceling some contracts at the same time.

Assume that $y \in A$ such that $\mu(y)=(b, s)$ (buyer $b$ and seller $s$ have a contract at $A$ ). Then, $b$ and $s$ can block allocation $A$ if there is a contract $x \in X \backslash A, \mu(x)=(b, s)$, such that $b$ and $s$ strictly prefer replacing $y$ by $x$ in their respective sets of contracts $A_{b}$ and $A_{s}$ while possibly canceling some contracts at the same time.

A buyer $b$ and a seller $s$ pairwise block an allocation $A$ if

(PB) there exists a contract $x \in X \backslash A, \mu(x)=(b, s)$, such that $x \in C_{b}(A \cup x)$ and $x \in C_{s}(A \cup x)$ - buyer $b$ and seller $s$ would like to implement contract $x$ while possibly canceling contracts in $A$.

An allocation $A$ is pairwise stable if it is individually rational (IR) and no buyer and seller can pairwise block it $[$ not $(\mathrm{PB})]$. By $P S(R) \subseteq \mathcal{A}$ we denote the set of pairwise stable allocations for matching market $R$.

Next, we introduce various notions of setwise stability.

\subsection{Strong Setwise Stability}

Let $B^{\prime} \subseteq B, S^{\prime} \subseteq S, N^{\prime}=B^{\prime} \cup S^{\prime}$, and assume that allocation $A$ is individually rational. We define strong setwise blocking of an allocation $A$ by the set of agents $N^{\prime}$ as follows.

Each member of the blocking coalition $N^{\prime}$ can add contracts with members of $N^{\prime}$ or replace existing contracts with members of $N^{\prime}$ while possibly canceling other contracts. If all members of $N^{\prime}$ can obtain a better set of contracts by adding, replacing, and/or canceling contracts as described above, then they can strongly setwise block allocation $A$.

A set of agents $N^{\prime}=B^{\prime} \cup S^{\prime}$ strongly setwise blocks an allocation $A$ if

(SSB) there exists a set of contracts $X^{\prime} \in \mathcal{A}$ such that

(1) for all $x \in X^{\prime} \backslash A, \mu(x) \in B^{\prime} \times S^{\prime}$

- new contracts are among the members of the blocking coalition only,

(2) for all $i \in N^{\prime}, X_{i}^{\prime} P_{i} A_{i}$

- all members of the blocking coalition receive a better set of contracts, and

(3) for all $j \in N \backslash N^{\prime}, X_{j}^{\prime} \subseteq A_{j}$

- agents outside the blocking coalition do not receive new contracts, but possibly some of their contracts are canceled by members of the blocking coalition. 
An allocation $A$ is strongly setwise stable if it is individually rational (IR) and no set of agents can strongly setwise block it [not (SSB)]. By $S S S(R) \subseteq \mathcal{A}$ we denote the set of strongly setwise stable allocations for matching market $R$.

Note that from the definition of strongly setwise blocking (SSB) we can easily construct a corresponding "blocking allocation $A^{\prime} \in \mathcal{A}$ " defined as follows:

(i) for all $i \in N^{\prime}, A_{i}^{\prime}=X_{i}^{\prime}$

- members of the blocking coalition receive their "blocking contract sets" $X_{i}^{\prime}$, and

(ii) for all $j \in N \backslash N^{\prime}$, $A_{j}^{\prime}=X_{j}^{\prime} \cup\left\{x \in A_{j} \mid\left[\mu(x)=(j, s)\right.\right.$ and $\left.s \in N \backslash N^{\prime}\right]$ or $\left[\mu(x)=(b, j)\right.$ and $\left.\left.b \in N \backslash N^{\prime}\right]\right\}$

- agents outside the blocking coalition receive all previous contracts with agents outside the blocking coalition and all previous contracts with the members of the blocking coalition that were not canceled.

If an allocation $A^{\prime}$ is such that some $N^{\prime}=B^{\prime} \cup S^{\prime}$ can strongly setwise block allocation $A$ with $X^{\prime}=\cup_{i \in N^{\prime}} A_{i}^{\prime}$, then we say that allocation $A$ is strongly setwise blocked via allocation $A^{\prime}$.

Our definition of strong setwise stability corresponds to Konishi and Ünver's (2006) group stability - defined for many-to-many matching markets. It is interesting to note that the verbal description of Sotomoyor's (1999, page 56, item (3)) setwise blocking coincides with our strong setwise stability.

The requirements of a strongly setwise block are minimal requirements for a blocking coalition to form. We next discuss two weaker setwise stability notions that are obtained by adding extra blocking conditions.

\subsection{Setwise Stability}

If we require that for all members of the blocking coalition the new set of contracts that is obtained by blocking (the new contracts with members of the blocking coalition and the old contracts that are kept with agents outside the blocking coalition) is preferred to the old set of contracts and individually rational, then we obtain the weaker notion of setwise blocking as, for instance, used in Echenique and Oviedo (2006).

A set of agents $N^{\prime}=B^{\prime} \cup S^{\prime}$ setwise blocks an allocation $A$ if

(SB) there exists a set of contracts $X^{\prime} \in \mathcal{A}$ such that

(1) for all $x \in X^{\prime} \backslash A, \mu(x) \in B^{\prime} \times S^{\prime}$

- new contracts are among the members of the blocking coalition only,

(2) for all $i \in N^{\prime}, X_{i}^{\prime} P_{i} A_{i}$ and $X_{i}^{\prime}=C_{i}\left(X_{i}^{\prime}\right)$,

- all members of the blocking coalition receive a better and individually rational set of contracts, and

(3) for all $j \in N \backslash N^{\prime}, X_{j}^{\prime} \subseteq A_{j}$

- agents outside the blocking coalition do not receive new contracts, but possibly some of their contracts are canceled by members of the blocking coalition.

An allocation $A$ is setwise stable if it is individually rational (IR) and no set of agents can setwise block it [not $(\mathrm{SB})]$. By $S S(R) \subseteq \mathcal{A}$ we denote the set of setwise stable allocations for matching market $R$.

Note that from the definition of setwise blocking (SB) we can similarly as in Section 3.2 construct a corresponding blocking allocation $A^{\prime} \in \mathcal{A}$ and define setwise blocking of allocation $A$ via allocation $A^{\prime}$. 
Our definition of setwise stability corresponds to Echenique and Oviedo's (2006) setwise stability. It is closely related to Konishi and Ünver's (2006) and Sotomoyor's (1999) setwise stability. The difference is that we only require individual rationality for the blocking coalition while Konishi and Ünver (2006) and Sotomoyor (1999) require that a setwise blocking results in an individually rational blocking allocation (hence individual rationality has to hold for all agents and not only the agents who block). For substitutable preferences these two notions of setwise stability coincide, but one can easily show that for general preferences our setwise stability is stronger than Konishi and Ünver's (2006) and Sotomoyor's (1999) setwise stability - our setwise blocking notion admits more setwise blockings and therefore in comparison fewer setwise stable allocations result. ${ }^{7}$

\subsection{Weak Setwise Stability}

Next, we further strengthen the setwise blocking assumption, and thereby weaken setwise stability, by requiring that all members of the blocking coalition obtain their best set of contracts among their original contracts and the new contracts of the blocking coalition.

A set of agents $N^{\prime}=B^{\prime} \cup S^{\prime}$ weakly setwise blocks an allocation $A$ if

(WSB) there exists a set of contracts $X^{\prime} \in \mathcal{A}$ such that

(1) for all $x \in X^{\prime} \backslash A, \mu(x) \in B^{\prime} \times S^{\prime}$

- new contracts are among the members of the blocking coalition only,

(2) for all $i \in N^{\prime}, X_{i}^{\prime} \neq A_{i}$ and $X_{i}^{\prime}=C_{i}\left(A \cup X^{\prime}\right)$,

- all members of the blocking coalition receive their best set of contracts among their original contracts and the new contracts of the blocking coalition, and

(3) for all $j \in N \backslash N^{\prime}, X_{j}^{\prime} \subseteq A_{j}$

- agents outside the blocking coalition do not receive new contracts, but possibly some of their contracts are canceled by members of the blocking coalition.

An allocation $A$ is weakly setwise stable if it is individually rational (IR) and no set of agents can weakly setwise block it [not (WSB)]. By $W S S(R) \subseteq \mathcal{A}$ we denote the set of weakly setwise stable allocations for matching market $R$.

Note that from the definition of weakly setwise blocking (WSB) we can similarly as in Section 3.2 construct a corresponding blocking allocation $A^{\prime} \in \mathcal{A}$ and define weakly setwise blocking of allocation $A$ via allocation $A^{\prime}$.

Weak setwise-stability is a new stability concept that, as we will see in Section 4.2 (Theorem 2), bridges the gap between pairwise stability and previously considered setwise stability notions.

\footnotetext{
${ }^{7}$ Note that the verbal description of Sotomoyor's 1999, page 56, item (3) setwise stability corresponds to our strong setwise stability property. However, in the formal definition (see Sotomoyor, 1999, Pages 59 and 60 , Definitions 1 and 2) Sotomayor includes individual rationality of the blocking allocation by requiring feasibility (which includes individual rationality).
} 


\section{Relations between Stability Notions}

\subsection{Stability in General}

We now show that on the unrestricted preference domain there is a set inclusion relation between all stability notions introduced so far. Furthermore, we provide a matching market for which all set inclusion relations are strict and a matching market without pairwise stable allocations.

\section{Theorem 1. The "Onion Structure of Stable Sets" - General Preferences}

(i) For all matching markets $R \in \mathcal{R}, S S S(R) \subseteq S S(R) \subseteq W S S(R) \subseteq P S(R)$.

(ii) There exist matching markets $R \in \mathcal{R}$ such that $S S S(R) \varsubsetneqq S S(R) \varsubsetneqq W S S(R) \varsubsetneqq P S(R)$.

(iii) There exist matching markets $R \in \mathcal{R}$ such that $P S(R)=\emptyset$.

Proof. (i) Consider matching market $R \in \mathcal{R}$.

By the definitions of strongly setwise blocking (SSB) and setwise blocking (SB) it is clear that any allocation $A$ that is setwise blocked is also strongly setwise blocked. Hence, $S S S(R) \subseteq S S(R)$.

Assume that allocation $A$ is weakly setwise blocked (WSB), i.e., there exists a coalition $N^{\prime}=B^{\prime} \cup S^{\prime}$ and a set contracts $X^{\prime} \in \mathcal{A}$ such that for all $x \in X^{\prime} \backslash A, \mu(x) \in B^{\prime} \times S^{\prime}$, for all $i \in N^{\prime}, X_{i}^{\prime} \neq A_{i}$ and $X_{i}^{\prime}=C_{i}\left(A \cup X^{\prime}\right)$, and for all $j \in N \backslash N^{\prime}, X_{j}^{\prime} \subseteq A_{j}$.

Note that $X_{i}^{\prime} \neq A_{i}$ and $X_{i}^{\prime}=C_{i}\left(A \cup X^{\prime}\right)$ imply that $X_{i}^{\prime} P_{i} A_{i}$. Furthermore, since $X_{i}^{\prime} \subseteq\left(A \cup X^{\prime}\right), X_{i}^{\prime}=C_{i}\left(A \cup X^{\prime}\right)$ implies $X_{i}^{\prime}=C_{i}\left(X_{i}^{\prime}\right)$. So, any allocation $A$ that is weakly setwise blocked is also setwise blocked. Hence, $S S(R) \subseteq W S S(R)$.

Next, assume that allocation $A$ is pairwise blocked (PB), i.e., there exists a contract $x \in X \backslash A$ such that $\mu(x)=(b, s)$ and $x \in C_{b}(A \cup x)$ and $x \in C_{s}(A \cup x)$. Define $B^{\prime}:=\{b\}$, $S^{\prime}:=\{s\}, N^{\prime}:=\{b, s\}, X_{b}^{\prime}:=C_{b}(A \cup x), X_{s}^{\prime}:=C_{b}(A \cup x)$, and $X^{\prime}:=X_{b}^{\prime} \cup X_{s}^{\prime}$. Then, $X^{\prime} \backslash A=\{x\}$, for $i \in N^{\prime}, X_{i}^{\prime}=C_{i}(A \cup x)$, and for all $j \in N \backslash N^{\prime}, x \notin X_{j}^{\prime}$ (and therefore $\left.X_{j}^{\prime} \subseteq A_{j}\right)$.

Thus, $X^{\prime} \subseteq X$ is such that for all $x \in X^{\prime} \backslash A, \mu(x) \in B^{\prime} \times S^{\prime}$, for all $i \in N^{\prime}, X_{i}^{\prime} \neq A_{i}$ and $X_{i}^{\prime}=C_{i}\left(A \cup X^{\prime}\right)$, and for all $j \in N \backslash N^{\prime}, X_{j}^{\prime} \subseteq A_{j}$ and $N^{\prime}$ weakly setwise blocks (WSB) allocation $A$. So, any allocation $A$ that is pairwise blocked is also weakly setwise blocked. Hence, $W S S(R) \subseteq P S(R)$.

(ii) With Example 1 we introduce a matching market $R \in \mathcal{R}$ such that $S S S(R) \varsubsetneqq S S(R) \varsubsetneqq$ $W S S(R) \varsubsetneqq P S(R)$.

(iii) With Example 2 we introduce a matching market $R \in \mathcal{R}$ such that $P S(R)=\emptyset$.

\section{Example 1. A "Stable Matching Onion" for General Preferences}

We consider a matching market with two buyers and two sellers and a set of contracts $X=$ $\{a, b, c, d, e, f, g, h, i, j, k, l, m, n\}$. Table 1 indicates the bilateral structure of contracts in $X$. Table 2 first lists agents' preferences in its columns, e.g., buyer $b_{1}$ 's preferences are such that ej $P_{b_{1}} m P_{b_{1}}$ im $P_{b_{1}}$ ij $P_{b_{1}}$ ef $P_{b_{1}}$ ab $P_{b_{1}} \emptyset P_{b_{1}} \ldots$, where “..." represents any ordering of the remaining feasible sets of contracts. Second, we list the following allocations for matching market $R$ in the rows of Table 2: $A=\{a, b, c, d\}, B=\{e, f, g, h\}, C=\{i, j, k, l\}, D=$ $\{i, m, n, l\}$, and $E=\{e, j\}$. Finally, Figure 1 illustrates that for matching market $R$ we have 
$S S S(R) \varsubsetneqq S S(R) \varsubsetneqq W S S(R) \varsubsetneqq P S(R)$ : it is easy to check that $I R(R)=\{A, B, C, E\}$. Furthermore,

- allocation $A$ is pairwise stable, but weakly setwise blocked via allocation $B$;

- allocation $B$ is weakly setwise stable, but setwise blocked via allocation $C$, which is not a weakly setwise block because $C_{b_{1}}(e, f, i, j)=e j \neq i j$;

- allocation $C$ is setwise stable, but strongly setwise blocked via allocation $D$, which is not a setwise block because $C_{b_{1}}(i, m)=m \neq i m$;

- allocation $E$ is strongly setwise stable.

\begin{tabular}{|l||c|c|c|c|c|c|c|}
\hline$x$ & $a$ & $b$ & $c$ & $d$ & $e$ & $f$ & $g$ \\
$\mu(x)$ & $\left(b_{1}, s_{1}\right)$ & $\left(b_{1}, s_{2}\right)$ & $\left(b_{2}, s_{1}\right)$ & $\left(b_{2}, s_{2}\right)$ & $\left(b_{1}, s_{1}\right)$ & $\left(b_{1}, s_{2}\right)$ & $\left(b_{2}, s_{1}\right)$ \\
\hline \hline$x$ & $h$ & $i$ & $j$ & $k$ & $l$ & $m$ & $n$ \\
$\mu(x)$ & $\left(b_{2}, s_{2}\right)$ & $\left(b_{1}, s_{1}\right)$ & $\left(b_{1}, s_{2}\right)$ & $\left(b_{2}, s_{1}\right)$ & $\left(b_{2}, s_{2}\right)$ & $\left(b_{1}, s_{2}\right)$ & $\left(b_{2}, s_{1}\right)$ \\
\hline
\end{tabular}

Table 1: Example 1 - the bilateral structure of contracts.

\begin{tabular}{|c||c|c||c|c|}
\hline allocation & buyer $b_{1}$ & buyer $b_{2}$ & seller $s_{1}$ & seller $s_{2}$ \\
\hline \hline$E$ & $e j$ & & & \\
& $m$ & $n$ & & \\
$D$ & $i m$ & $n l$ & $i n$ & $m l$ \\
$C$ & $i j$ & $k l$ & $i k$ & $j l$ \\
$B$ & $e f$ & $g h$ & $e g$ & $f h$ \\
$A$ & $a b$ & $c d$ & $a c$ & $b d$ \\
$E$ & & & $e$ & $j$ \\
& $\emptyset$ & $\emptyset$ & $\emptyset$ & $\emptyset$ \\
& $\ldots$ & $\ldots$ & $\ldots$ & $\ldots$ \\
\hline
\end{tabular}

Table 2: Example 1 - preferences and allocations $A, B, C, D, E$. 


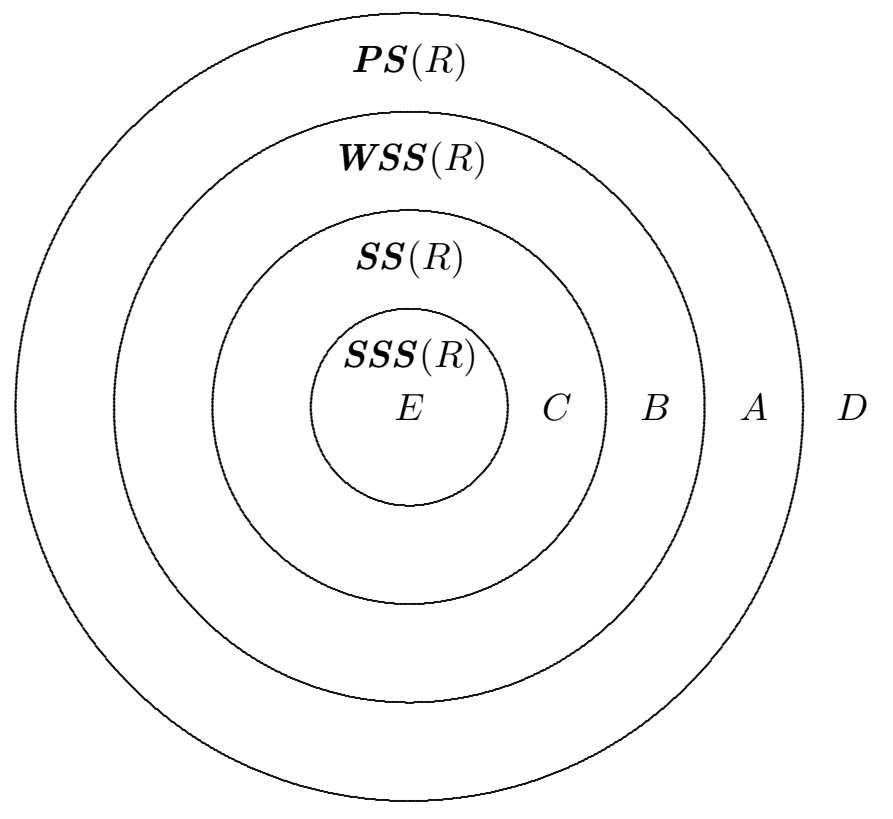

Figure 1: Example 1 - a "stable matching onion" and allocations $A, B, C, D, E$.

\section{Example 2. An Empty Set of Pairwise Stable Matchings}

We consider a matching market with three buyers and three sellers and a set of contracts $X=\{a, b, c, d, e, f\}$. Table 3 indicates the bilateral structure of contracts in $X$. Table 4 lists agents' preferences in its columns. To check that there exists no pairwise stable allocation, we start with the observation that no individually rational allocation at which a buyer has only one contract can be pairwise stable. To see this note that any buyer who has just one contract would be strictly better off by signing another one with another seller. The respective seller can then get his top choice (possibly by canceling another contract), and hence prefers to sign as well. On the other hand, no allocation at which a buyer signs two contracts can be pairwise stable. To see this note that whenever one buyer signs two contracts, no other buyer can contract with more than one seller to maintain individual rationality and feasibility of the allocation. But if a buyer can not sign a single contract in any pairwise stable allocation, this implies that all other buyers and one seller are unmatched. But then there is always a buyer and a seller who can improve upon the status quo by signing a contract. Hence, no allocation can be pairwise stable.

\begin{tabular}{|l||c|c|c|c|c|c|}
\hline$x$ & $a$ & $b$ & $c$ & $d$ & $e$ & $f$ \\
$\mu(x)$ & $\left(b_{1}, s_{1}\right)$ & $\left(b_{1}, s_{2}\right)$ & $\left(b_{2}, s_{2}\right)$ & $\left(b_{2}, s_{3}\right)$ & $\left(b_{3}, s_{3}\right)$ & $\left(b_{3}, s_{1}\right)$ \\
\hline
\end{tabular}

Table 3: Example 2 - the bilateral structure of contracts. 


\begin{tabular}{|c|c|c||c|c|c|}
\hline buyer $b_{1}$ & buyer $b_{2}$ & buyer $b_{3}$ & seller $s_{1}$ & seller $s_{2}$ & seller $s_{3}$ \\
\hline \hline$a b$ & $c d$ & $e f$ & $a$ & $c$ & $e$ \\
$b$ & $d$ & $f$ & $f$ & $b$ & $d$ \\
$\emptyset$ & $\emptyset$ & $\emptyset$ & $\emptyset$ & $\emptyset$ & $\emptyset$ \\
$\ldots$ & $\ldots$ & $\ldots$ & $\ldots$ & $\ldots$ & $\ldots$ \\
\hline
\end{tabular}

Table 4: Example 2 - preferences.

\subsection{Stability and Substitutability}

In his seminal paper Roth (1984b) proved that the set of pairwise stable matchings is nonempty for substitutable preferences. Here, we prove that weak setwise stability and pairwise stability coincide. Hence, for substitutable preferences we have found a setwise blocking concept that induces a nonempty stable set. ${ }^{8}$ Furthermore, we provide a matching market with substitutable preferences for which the remaining set inclusion relations are strict and a matching market with substitutable preferences without setwise stable allocations.

\section{Theorem 2. The "Onion Structure of Stable Sets" - Substitutable Preferences}

(i) For matching markets with substitutable preferences an allocation is weakly setwise stable if and only if it is pairwise stable.

(ii) For matching markets with substitutable preferences the set of pairwise (weakly setwise) stable matchings is nonempty.

Hence, (i) and (ii) imply that for all matching markets with substitutable preferences $R \in \mathcal{R}$,

$$
S S S(R) \subseteq S S(R) \subseteq W S S(R)=P S(R) \neq \emptyset .
$$

(iii) There exist matching markets with substitutable preferences $R \in \mathcal{R}$ such that $S S S(R) \varsubsetneqq$ $S S(R)$ and $S S(R) \varsubsetneqq W S S(R)=P S(R)$.

(iv) There exist matching markets with substitutable preferences $R \in \mathcal{R}$ such that $S S(R)=\emptyset$.

Proof. (i) Let $R$ be substitutable. By Theorem 1 (i), $W S S(R) \subseteq P S(R)$. It remains to prove that $P S(R) \subseteq W S S(R)$.

Assume that allocation $A$ is weakly setwise blocked (WSB), i.e., there exists a coalition $N^{\prime}=B^{\prime} \cup S^{\prime}$ and a set of contracts $X^{\prime} \in \mathcal{A}$ such that for all $x \in X^{\prime} \backslash A, \mu(x) \in B^{\prime} \times S^{\prime}$, for all $i \in N^{\prime}, X_{i}^{\prime} \neq A_{i}$ and $X_{i}^{\prime}=C_{i}\left(A \cup X^{\prime}\right)$, and for all $j \in N \backslash N^{\prime}, X_{j}^{\prime} \subseteq A_{j}$. Then, there exist $b \in B^{\prime}, s \in S^{\prime}$, and $x \in X^{\prime} \backslash A$ such that $\mu(x)=(b, s)$.

Let $i \in\{b, s\}$ and assume that $x \notin C_{i}(A \cup x)$ or, equivalently, $x \in N C_{i}(A \cup x)$. Note that $(A \cup x) \subseteq\left(A \cup X_{i}^{\prime}\right)$. Then, by substitutability (SUB'), $x \in N C_{i}\left(A \cup X_{i}^{\prime}\right)$. But then, since $x \in X_{i}^{\prime}, X_{i}^{\prime} \neq C_{i}\left(A \cup X_{i}^{\prime}\right)$; a contradiction. Thus, $x \in C_{b}(A \cup x)$ and $x \in C_{s}(A \cup x)$ and allocation $A$ is pairwise blocked (PB). So, any allocation $A$ that is weakly setwise blocked is also pairwise blocked. Hence, $P S(R) \subseteq W S S(R)$.

\footnotetext{
${ }^{8}$ For many-to-many matching markets with strongly substitutable preferences Echenique and Oviedo (2006) prove that the set of setwise stable matchings is nonempty. We extend this result to many-to-many matching markets with contracts in Section 3.2.
} 
Note that substitutability is a crucial assumption for our proof of the identity of $W S S(R)$ and $P S(R)$. A simple example of a situation where $W S S(R) \neq P S(R)$ is Example 3.

(ii) Roth (1984b, Theorem 1) proved for many-to-many matching markets with contracts and substitutable preferences that the set of pairwise stable matchings is nonempty.

(iii) With Example 4 we introduce a matching market with substitutable preferences $R \in$ $\mathcal{R}$ such that $S S S(R) \varsubsetneqq S S(R)$. With Example 5 we introduce a matching market with substitutable preferences $R \in \mathcal{R}$ such that $S S(R) \varsubsetneqq W S S(R)=P S(R)$.

(iv) Blair (1988, Example 2.6) presented a matching market $R$ with substitutable preferences (without contracts) to demonstrate that the core of a many-to-many matching market with substitutable preferences can be empty. It is easy to check that for this example (see also Roth and Sotomayor, 1990, Example 6.9), $S S(R)=\emptyset$.

Example 3. $P S(R) \neq W S S(R)$ if preferences of one agent are not substitutable.

We consider a matching market with two buyers and two sellers and a set of contracts $X=$ $\{a, b, c\}$. Table 5 indicates the bilateral structure of contracts in $X$. Table 6 first lists agents' preferences in its columns. Note that $b_{1}$ 's preferences are not substitutable while all other agent's preferences are. Second, we list the following allocations for matching market $R$ in the rows of Table 6: $A=\{c\}$ and $B=\{a, b\}$. Obviously, allocation $A$ is pairwise stable. However, it can be weakly setwise blocked by $\left\{b_{1}, s_{1}, s_{2}\right\}$ who agree upon allocation $B$. Hence, $P S(R)=\{A, B\} \neq\{B\}=W S S(R)$.

\begin{tabular}{|l||c|c|c|}
\hline$x$ & $a$ & $b$ & $c$ \\
$\mu(x)$ & $\left(b_{1}, s_{1}\right)$ & $\left(b_{1}, s_{2}\right)$ & $\left(b_{2}, s_{1}\right)$ \\
\hline
\end{tabular}

Table 5: Example 3 - the bilateral structure of contracts.

\begin{tabular}{|c||c|c||c|c|}
\hline allocation & buyer $b_{1}$ & buyer $b_{2}$ & seller $s_{1}$ & seller $s_{2}$ \\
\hline \hline $\mathrm{B}$ & $a b$ & & $a$ & $b$ \\
$\mathrm{~A}$ & $\emptyset$ & $c$ & $c$ & $\emptyset$ \\
& $a$ & $\emptyset$ & $\emptyset$ & \\
& $b$ & & $a c$ & \\
\hline
\end{tabular}

Table 6: Example 3 - preferences and allocations $A$ and $B$.

Example 4. $S S S(R) \subsetneq S S(R)$ for substitutable preferences.

We consider a matching market with two buyers and two sellers and a set of contracts $X=$ $\{a, b, c, d, e, f\}$. Table 7 indicates the bilateral structure of contracts in $X$. Table 8 first lists agents' substitutable preferences in its columns. Second, we list the following allocations for matching market $R$ in the rows of Table 8: $A=\{a, b\}, B=\{c, d, e, f\}$, and $C=$ $\{c, f\}$. Finally, Figure 2 illustrates that for matching market $R$ we have $S S S(R) \varsubsetneqq S S(R)=$ $W S S(R)=P S(R)$. To be specific,

- allocation $A$ is pairwise and (weakly) setwise stable, but strongly setwise blocked via allocation $B$, which is not a setwise block because $C_{b_{1}}(c, d)=c$;

- allocation $C$ is strongly setwise stable. 


\begin{tabular}{|l||c|c|c|c|c|c|}
\hline$x$ & $a$ & $b$ & $c$ & $d$ & $e$ & $f$ \\
$\mu(x)$ & $\left(b_{1}, s_{1}\right)$ & $\left(b_{2}, s_{2}\right)$ & $\left(b_{1}, s_{1}\right)$ & $\left(b_{1}, s_{2}\right)$ & $\left(b_{2}, s_{1}\right)$ & $\left(b_{2}, s_{2}\right)$ \\
\hline
\end{tabular}

Table 7: Example 4 - the bilateral structure of contracts.

\begin{tabular}{|c||c|c||c|c|}
\hline allocation & buyer $b_{1}$ & buyer $b_{2}$ & seller $s_{1}$ & seller $s_{2}$ \\
\hline \hline$C$ & $c$ & $f$ & & \\
& & & $e$ & $d$ \\
$B$ & $c d$ & $e f$ & $c e$ & $d f$ \\
$A$ & $a$ & $b$ & $a$ & $b$ \\
& $d$ & $e$ & & \\
$C$ & & & $c$ & $f$ \\
& $a d$ & $e b$ & $a e$ & $b d$ \\
& $\emptyset$ & $\emptyset$ & $\emptyset$ & $\emptyset$ \\
\hline
\end{tabular}

Table 8: Example 4 - preferences and allocations $A, B$, and $C$.

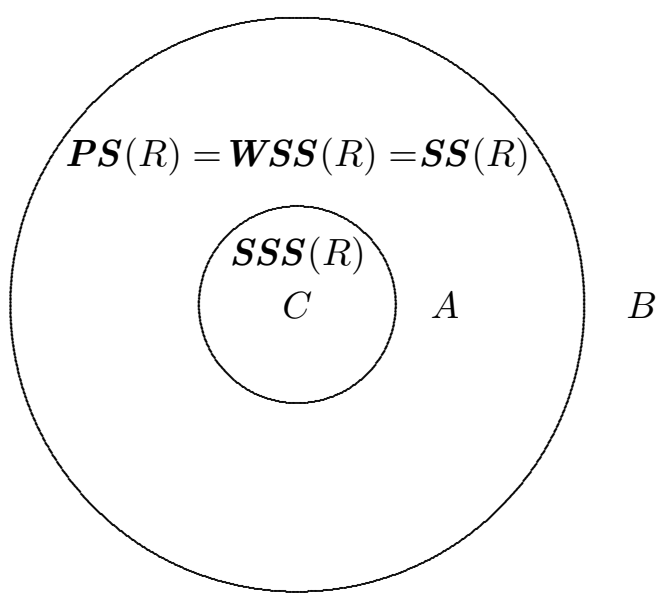

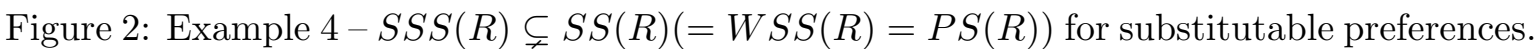


Example 5. $S S(R) \subsetneq W S S(R)=P S(R)$ for substitutable preferences

We consider a matching market with two buyers and two sellers and a set of contracts $X=$ $\{a, b, c, d, e, f, g, h\}$. Table 9 indicates the bilateral structure of contracts in $X$. Table 10 first lists agents' substitutable preferences in its columns. Second, we list the following allocations for matching market $R$ in the rows of Table 10: $A=\{a, b, c, d\}$ and $B=\{e, f, g, h\}$. Finally, Figure 3 illustrates that for matching market $R$ we have $S S(R) \varsubsetneqq W S S(R)=P S(R)$. To be specific,

- allocation $A$ is pairwise and weakly setwise stable but not setwise stable ( $B$ setwise blocks allocation $A$ but is not a weakly setwise block because e.g., $C_{b_{1}}($ abef $\left.)=a f \neq e f\right)$.

- allocation $B$ is setwise stable.

\begin{tabular}{|l||c|c|c|c|c|c|c|c|}
\hline$x$ & $a$ & $b$ & $c$ & $d$ & $e$ & $f$ & $g$ & $h$ \\
$\mu(x)$ & $\left(b_{1}, s_{1}\right)$ & $\left(b_{1}, s_{2}\right)$ & $\left(b_{2}, s_{1}\right)$ & $\left(b_{2}, s_{2}\right)$ & $\left(b_{1}, s_{1}\right)$ & $\left(b_{1}, s_{2}\right)$ & $\left(b_{2}, s_{1}\right)$ & $\left(b_{2}, s_{2}\right)$ \\
\hline
\end{tabular}

Table 9: Example 5 - the bilateral structure of contracts.

\begin{tabular}{|c||c|c||c|c|}
\hline allocation & buyer $b_{1}$ & buyer $b_{2}$ & seller $s_{1}$ & seller $s_{2}$ \\
\hline \hline \multirow{3}{*}{$B$} & $a f$ & $d g$ & $c e$ & $b h$ \\
$A$ & $e f$ & $g h$ & $e g$ & $f h$ \\
& $a b$ & $c d$ & $a c$ & $b d$ \\
& $b e$ & $c h$ & $a g$ & $d f$ \\
& $f$ & $g$ & $e$ & $h$ \\
& $b$ & $c$ & $a$ & $d$ \\
& $a$ & $d$ & $c$ & $b$ \\
& $e$ & $h$ & $g$ & $f$ \\
& $\emptyset$ & $\emptyset$ & $\emptyset$ & $\emptyset$ \\
\hline
\end{tabular}

Table 10: Example 5 - substitutable preferences, and allocations $A$ and $B$.

\section{Remark 1. Setwise Stability in Roth (1984b)}

Theorem 1 in Roth (1984b) states that for many-to-many matching markets with contracts and substitutable preferences the set of (setwise) stable matchings is nonempty. Later there were some claims that the result shown in Roth (1984b) was not correct and only holds for the set of pairwise stable matchings (see for instance Roth and Sotomayor, 1990, page 185, lines 6-9). In the Appendix we demonstrate that Roth (1984b, Theorem 1) was correct as stated. The only confusion that arose is concerning the exact definition of (setwise) stability according to Roth's (1984b) definition his result is correct, but his notion of (setwise) stability differs from the definitions as introduced in Section 3. 


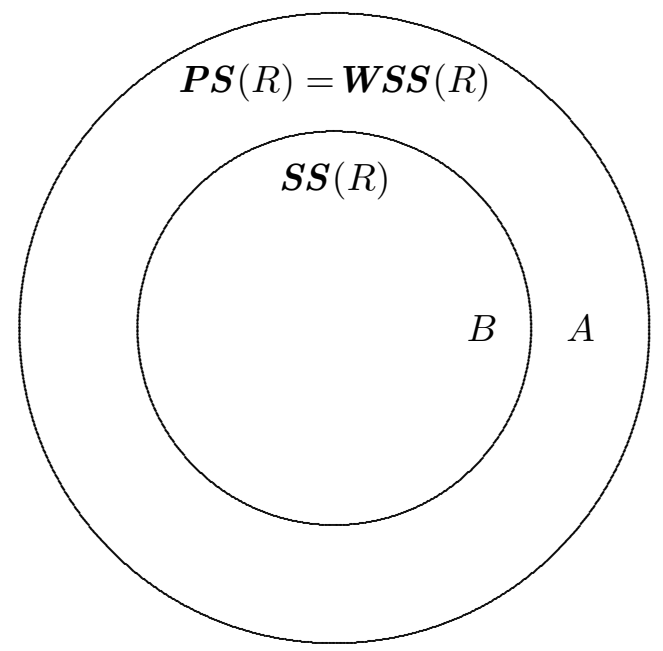

Figure 3: Example $5-S S(R) \subsetneq W S S(R)=P S(R)$ for substitutable preferences.

\subsection{Stability and Strong Substitutability}

For strongly substitutable preferences setwise stability, weak setwise stability, and pairwise stability coincide. Echenique and Oviedo (2006) proved this result for many-to-many matching markets. Since we consider many-to-many matching markets with contracts, our result implies theirs as a special case. Since strong substitutability implies substitutability, Theorem 2 implies that the set of setwise stable matchings is nonempty. Furthermore, we provide a matching market with strongly substitutable preferences for which the set of strongly setwise stable matchings is a strict subset of the set of setwise stable matchings and a matching market with strongly substitutable preferences without strongly setwise stable allocations.

\section{Theorem 3. The "Onion Structure of Stable Sets" - Strongly Substitutable Pref-} erences

(i) For matching markets with strongly substitutable preferences an allocation is setwise stable if and only if it is pairwise stable. Hence, for all matching markets with strongly substitutable preferences $R \in \mathcal{R}, S S(R)=W S S(R)=P S(R) \neq \emptyset$.

(ii) There exist matching markets with strongly substitutable preferences $R \in \mathcal{R}$ such that $S S S(R) \varsubsetneqq S S(R)$.

(iii) There exist matching markets with strongly substitutable preferences $R \in \mathcal{R}$ such that $S S S(R)=\emptyset$.

Proof. (i) By Theorem 1, $S S(R) \subseteq W S S(R) \subseteq P S(R)$. We complete the proof by showing that $P S(R) \subseteq S S(R)$.

Assume that allocation $A$ is setwise blocked (SB), i.e., there exists a coalition $N^{\prime}=B^{\prime} \cup S^{\prime}$ and a set of contracts $X^{\prime} \in \mathcal{A}$ such that for all $x \in X^{\prime} \backslash A, \mu(x) \in B^{\prime} \times S^{\prime}$, for all $i \in N^{\prime}$, $X_{i}^{\prime} P_{i} A_{i}$ and $X_{i}^{\prime}=C_{i}\left(X_{i}^{\prime}\right)$, and for all $j \in N \backslash N^{\prime}, X_{j}^{\prime} \subseteq A_{j}$. Then, there exist $b \in B^{\prime}, s \in S^{\prime}$, and $x \in X^{\prime} \backslash A$ such that $\mu(x)=(b, s)$. Note that $x \in C_{i}\left(X_{i}^{\prime}\right)$.

Let $i \in\{b, s\}$ and assume that $x \notin C_{i}(A \cup x)$. Hence, $X_{i}^{\prime} P_{i} C_{i}\left(A_{i} \cup x\right)$. Then, by strong substitutability (SSUB), $\left(A_{i} \cup x\right) \cap C_{i}\left(X^{\prime}\right) \subseteq C_{i}\left(A_{i} \cup x\right)$. Since $x \in(A \cup x) \cap C_{i}\left(X^{\prime}\right)$, $x \in C_{i}(A \cup x)$; a contradiction. Thus, $x \in C_{b}(A \cup x)$ and $x \in C_{s}(A \cup x)$ and allocation $A$ is 
pairwise blocked (PB). So, any allocation $A$ that is setwise blocked is also pairwise blocked. Hence, $P S(R) \subseteq S S(R)$.

Note that strong substitutability is a crucial assumption for our proof of the identity of $S S(R)$ and $P S(R)$. A simple example (with substitutable preferences) of a situation where $S S(R) \neq P S(R)$ is Blair (1988, Example 2.6).

(ii) Preferences in Example 4 are actually strongly substitutable. Hence, this example is a matching market with strongly substitutable preferences $R \in \mathcal{R}$ such that $S S S(R) \varsubsetneqq S S(R)$.

(iii) With Example 6 we introduce a matching market with strongly substitutable preferences $R \in \mathcal{R}$ such that $S S S(R)=\emptyset$.

Example 6. Strongly Substitutable Preferences and an Empty Set of Strongly Setwise Stable Matchings

We consider a matching market with three buyers and three sellers and a set of contracts $X=\{a, b, c, d, e, f, h, i\}$. Table 11 indicates the bilateral structure of contracts in $X$. Table 12 lists agents' strongly substitutable preferences in its columns. To check that there exists no strongly setwise stable allocation, we start with the observation that no allocation that assigns all agents a set of contracts below their respective (complete) set of contracts (i.e., allocation $X$ ) can be strongly setwise stable (agents can use all their contracts to block). Note that for instance $A=\{a, d, g\}$ is pairwise (and weakly setwise) stable; the only feasible blocking $(X)$ is not individually rational. Next, no allocation that assigns an agent her (complete) set of contracts can be strongly setwise stable since any such agent would be better off by canceling one of the contracts. Thus, at least one agent has to receive her best set of contracts. For instance, assume that there exists a strongly setwise stable allocation $B$ at which buyer $b_{1}$ obtains contracts $a$ and $b$. Then, seller $s_{2}$ has to obtain only contract $b$ (no other contract set containing $b$ is individually rational for seller $s_{2}$ ). But then agents $b_{2}$ and $s_{2}$ could block allocation $B$ using contract $d$. So, no such strongly setwise stable allocation $B$ at which buyer $b_{1}$ obtains her best set of contracts exists. Similarly, we can prove that no strongly setwise stable allocation $B$ at which agent $i \in\left\{b_{2}, b_{3}, s_{1}, s_{2}, s_{3}\right\}$ obtains her best set of contracts exists. Hence, no allocation can be strongly setwise stable.

\begin{tabular}{|l||c|c|c|c|c|}
\hline $\begin{array}{l}x \\
\mu(x)\end{array}$ & $\begin{array}{c}a \\
\left(b_{1}, s_{1}\right)\end{array}$ & $\begin{array}{c}b \\
\left(b_{1}, s_{2}\right)\end{array}$ & $\begin{array}{c}c \\
\left(b_{1}, s_{3}\right)\end{array}$ & $\begin{array}{c}d \\
\left(b_{2}, s_{2}\right)\end{array}$ & $\begin{array}{c}e \\
\left(b_{2}, s_{3}\right)\end{array}$ \\
\hline \hline$x$ & $f$ & $g$ & $h$ & $i$ & \\
$\mu(x)$ & $\left(b_{2}, s_{1}\right)$ & $\left(b_{3}, s_{3}\right)$ & $\left(b_{3}, s_{1}\right)$ & $\left(b_{3}, s_{2}\right)$ & \\
\hline
\end{tabular}

Table 11: Example 6 - the bilateral structure of contracts. 


\begin{tabular}{|c|c|c||c|c|c|}
\hline buyer $b_{1}$ & buyer $b_{2}$ & buyer $b_{3}$ & seller $s_{1}$ & seller $s_{2}$ & seller $s_{3}$ \\
\hline \hline$a b$ & $d e$ & $g h$ & $a f$ & $d i$ & $c g$ \\
$a b c$ & $d e f$ & $g h i$ & $a f h$ & $b d i$ & $c g e$ \\
$a$ & $d$ & $g$ & $a$ & $d$ & $g$ \\
$a c$ & $d f$ & $g i$ & $a h$ & $b d$ & $e g$ \\
$b c$ & $e f$ & $h i$ & $f h$ & $b i$ & $c e$ \\
$b$ & $e$ & $h$ & $f$ & $i$ & $c$ \\
$c$ & $f$ & $i$ & $h$ & $b$ & $e$ \\
$\emptyset$ & $\emptyset$ & $\emptyset$ & $\emptyset$ & $\emptyset$ & $\emptyset$ \\
\hline
\end{tabular}

Table 12: Example 6 - preferences.

\section{References}

Blair, C. (1988): "The Lattice Structure of the Set of Stable Matchings with Multiple Partners." Mathematics of Operations Research, 13: 619-628.

Echenique, F. and Oviedo, J. (2006): "A Theory of Stability in Many-to-Many Matching Markets." Theoretical Economics, 1: 233-273.

Gale, D. and Shapley, L. (1962): "College Admissions and the Stability of Marriage." American Mathematical Monthly, 69: 9-15.

Haake, C.-J. and Klaus, B. (2005): "Monotonicity and Nash Implementation in Matching Markets with Contracts." METEOR RM/05/029.

Hatfield, J. W. and Milgrom, P. (2005): "Matching with Contracts." American Economic Review, 95(4): 913-935.

Kelso, A. S. and Crawford, V. P. (1982): "Job Matching, Coalition Formation, and Gross Substitutes." Econometrica, 6: 1483-1504.

Konishi, H. and Ünver, M. U. (2006): "Credible Group Stability in Many-to-Many Matching Problems." Journal of Economic Theory, 129: 57-80.

Milgrom, P. (2004): Putting Auction Theory to Work. Cambridge University Press, Cambridge.

Roth, A. E. (1982): "The Economics of Matching: Stability and Incentives." Mathematics of Operations Research, 7: 617-628.

Roth, A. E. (1984a): "The Evolution of the Labor Market for Medical Interns and Residents: a Case Study in Game Theory." Journal of Political Economy, 92: 991-1016.

Roth, A. E. (1984b): "Stability and Polarization of Interests in Job Matching." Econometrica, 52: $47-57$.

Roth, A. E. (1985): "The College Admissions Problem Is Not Equivalent to the Marriage Problem." Journal of Economic Theory, 63(2): 277-288. 
Roth, A. E. (1991): "A Natural Experiment in the Organization of Entry-Level Labor Markets: Regional Markets for New Physicians and Surgeons in the United Kingdom." American Economic Review, 81: 415-440.

Roth, A. E. and Sotomayor, M. A. (1990): Two-Sided Matching: A Study in Game-Theoretic Modeling and Analysis. Cambridge University Press, Cambridge.

Roth, A. E. and Xing, X. (1994): "Jumping the Gun: Imperfections and Institutions Related to the Timing of Market Transactions." American Economic Review, 84: 992-1044.

Sotomoyor, M. (1999): "Three Remarks on the Many-to-Many Stable Matching Problem." Mathematical Social Sciences, 38: 55-70.

\section{Appendix}

\section{Roth's (1984b) (Setwise) Stability}

Roth's (1984b) definition of (setwise) stability does not coincide with the notions introduced in Section 3. Roth also strengthened the (setwise) blocking condition and thereby weakened (setwise) stability. He required that all members of the blocking coalition obtain a subset of their best set of contracts among their original contracts and the new contracts of the blocking coalition.

A set of agents $N^{\prime}=B^{\prime} \cup S^{\prime}$ Roth blocks an allocation $A$ if

(RSB) there exists a set of contracts $Y^{\prime} \in \mathcal{A}$ such that

(1) $\cup_{x \in Y^{\prime}} \mu(x)=B^{\prime} \times S^{\prime}$ and

(2) for all $i \in N^{\prime}, Y_{i}^{\prime} \subseteq C_{i}\left(A \cup Y^{\prime}\right) P_{i} A_{i}$.

An allocation $A$ is Roth stable if it is individually rational (IR) and no set of agents can Roth block allocation $A$ [not (RSB)]. By $R S S(R) \subseteq \mathcal{A}$ we denote the set of Roth stable allocations for matching market $R$.

We now show that there is a set inclusion relation between all stability notions introduced so far if instead of setwise stability we consider Roth stability. On the other hand, Roth stability and setwise stability are not logically related. Hence, Roth (1984b) and Sotomoyor (1999) refer to different stability notions when they use the term (setwise) stability.

\section{Proposition 1. Roth Stability and General Preferences}

(i) For all matching markets $R \in \mathcal{R}, S S S(R) \subseteq R S S(R) \subseteq W S S(R) \subseteq P S(R)$.

(ii) There exist matching markets $R \in \mathcal{R}$ such that $S S(R) \subsetneq R S S(R)$.

(iii) There exist matching markets $R \in \mathcal{R}$ such that $R S S(R) \subsetneq S S(R)$.

Proof. (i) Consider matching market $R \in \mathcal{R}$.

By the definitions of strongly setwise blocking (SSB) and Roth setwise blocking (RSB) it is clear that any allocation $A$ that is Roth setwise blocked is also strongly setwise blocked. Hence, $S S S(R) \subseteq R S S(R)$. 
Assume that allocation $A$ is weakly setwise blocked (WSB), i.e., there exists a coalition $N^{\prime}=B^{\prime} \cup S^{\prime}$ and a set contracts $X^{\prime} \in \mathcal{A}$ such that for all $x \in X^{\prime} \backslash A, \mu(x) \in B^{\prime} \times S^{\prime}$, for all $i \in N^{\prime}, X_{i}^{\prime} \neq A_{i}$ and $X_{i}^{\prime}=C_{i}\left(A \cup X^{\prime}\right)$, and for all $j \in N \backslash N^{\prime}, X_{j}^{\prime} \subseteq A_{j}$. Let $Y^{\prime}:=X^{\prime} \backslash A$. Then, for all $i \in N^{\prime}, Y_{i}^{\prime} \subseteq C_{i}\left(A \cup Y^{\prime}\right) P_{i} A_{i}$. So, any allocation $A$ that is weakly setwise blocked is also Roth setwise blocked. Hence, $R S S(R) \subseteq W S S(R)$.

(ii) For Example $5 S S(R) \subsetneq W S S(R)=R S S(R)$ (since preferences in Example 5 are substitutable, $W S S(R)=R S S(R)$ follows from Proposition 2).

(iii) With Example 7 we introduce a matching market $R \in \mathcal{R}$ such that $R S S(R) \subsetneq S S(R)$.

\section{Example 7. $R S S(R) \subsetneq S S(R)$ for General Preferences}

We consider a matching market with three buyers and two sellers and a set of contracts $X=$ $\{a, b, c, d, e, f, g, h, i, j\}$. Table 13 indicates the bilateral structure of contracts in $X$. Table 14 first lists agents' preferences in its columns. Second, we indicate the unique individually rational allocation for matching market $R$ in the second row of Table 14: $A=\{a, b, d, e, i, j\}$. Obviously, allocation $A$ is setwise stable and $S S(R)=\{A\}$. However, it can be Roth setwise blocked by $\left\{b_{1}, b_{2}, b_{3}, s_{1}, s_{2}\right\}$ and $Y^{\prime}=\{c, f, g, h\}$. Hence, $R S S(R)=\emptyset \subsetneq\{A\}=S S(R)$. Note, that the Roth block $Y^{\prime}$ is not an individually rational allocation.

\begin{tabular}{|l||c|c|c|c|c|}
\hline$x$ & $a$ & $b$ & $c$ & $d$ & $e$ \\
$\mu(x)$ & $\left(b_{1}, s_{1}\right)$ & $\left(b_{1}, s_{2}\right)$ & $\left(b_{1}, s_{2}\right)$ & $\left(b_{2}, s_{1}\right)$ & $\left(b_{2}, s_{2}\right)$ \\
\hline \hline$x$ & $f$ & $g$ & $h$ & $i$ & $j$ \\
$\mu(x)$ & $\left(b_{2}, s_{1}\right)$ & $\left(b_{3}, s_{1}\right)$ & $\left(b_{3}, s_{2}\right)$ & $\left(b_{3}, s_{1}\right)$ & $\left(b_{3}, s_{1}\right)$ \\
\hline
\end{tabular}

Table 13: Example 7 - the bilateral structure of contracts.

\begin{tabular}{|c||c|c|c||c|c|}
\hline allocation & buyer $b_{1}$ & buyer $b_{2}$ & buyer $b_{3}$ & seller $s_{1}$ & seller $s_{2}$ \\
\hline \hline \multirow{4}{*}{$\mathrm{A}$} & $c$ & $f$ & $g h$ & $a f g$ & $c e h$ \\
& $a b$ & $d e$ & $i j$ & $a d i$ & $b e j$ \\
& $\emptyset$ & $\emptyset$ & $\emptyset$ & $\emptyset$ & $\emptyset$ \\
& $\ldots$ & $\ldots$ & $\ldots$ & $\ldots$ & $\ldots$ \\
\hline
\end{tabular}

Table 14: Example 7 - preferences and allocation $A$.

Finally, we prove that for substitutable preferences Roth setwise stability and pairwise stability coincide. Moreover, we show that both sets are nonempty. Roth (1984b, Lemma 2 and Theorem 1) already proved these results, but then there were some statements that the equality of Roth setwise stable sets and pairwise stable sets is false, e.g., Roth and Sotomayor (1990, page 185, lines 7-9) state that in Roth (1984b)

readers [...] are advised that they need to substitute "pairwise stable" for "stable" in the results concerning many-to-many matching.

and Sotomoyor (1999, page 57, lines 11-13) claims that 
For the case with substitutable preferences the existence theorem of Roth (1984) proves the existence of pairwise-stable matchings and not, as the author claims, that of stable matchings.

To a certain extend Roth (1984b, Lemma 2 and Theorem 1) is correct and the above statements are also correct. The reason for the confusion is that two different notions of stability are compared: Roth (1984b) used what we call Roth setwise stability and Roth and Sotomayor (1990) and Sotomoyor (1999) both refer to what we call setwise stability.

\section{Proposition 2. Roth Stability and Substitutability}

(i) For matching markets with substitutable preferences an allocation is Roth setwise stable if and only if it is pairwise stable.

(ii) For matching markets with substitutable preferences the set of Roth setwise stable matchings is nonempty.

Hence, (i) and (ii) imply that for all matching markets with substitutable preferences $R \in \mathcal{R}$,

$$
S S S(R) \subseteq S S(R) \subseteq R S S(R)=W S S(R)=P S(R) \neq \emptyset .
$$

Proof. (i) Let $R$ be substitutable. By Proposition 1 (i), $R S S(R) \subseteq P S(R)$. It remains to prove that $P S(R) \subseteq R S S(R)$.

Assume that allocation $A$ is Roth setwise blocked, i.e., there exists a coalition $N^{\prime}=$ $B^{\prime} \cup S^{\prime}$ and a set of contracts $Y^{\prime} \in \mathcal{A}$ such that $\cup_{x \in Y^{\prime}} \mu(x)=B^{\prime} \times S^{\prime}$ and for all $i \in N^{\prime}$, $Y_{i}^{\prime} \subseteq C_{i}\left(A \cup Y^{\prime}\right) P_{i} A_{i}$. Then, there exist $b \in B^{\prime}, s \in S^{\prime}$, and $x \in Y^{\prime} \backslash A$ such that $\mu(x)=(b, s)$.

Let $i \in\{b, s\}$ and assume that $x \notin C_{i}(A \cup x)$ or, equivalently, $x \in N C_{i}(A \cup x)$. Note that $A \cup x \subseteq A \cup Y_{i}^{\prime}$. Then, by substitutability (SUB'), $x \in N C_{i}\left(A \cup Y_{i}^{\prime}\right)$. But then, since $x \in Y_{i}^{\prime}$, $Y_{i}^{\prime} \nsubseteq C_{i}\left(A \cup Y_{i}^{\prime}\right)$; a contradiction. Thus, $x \in C_{b}(A \cup x)$ and $x \in C_{s}(A \cup x)$ and allocation $A$ is pairwise blocked (PB). So, any allocation $A$ that is Roth setwise blocked is also pairwise blocked. Hence, $P S(R) \subseteq R S S(R)$.

(ii) By (i), for all $R \in \mathcal{R}, R S S(R)=W S S(R)$. By Theorem $2, W S S(R)=P S(R) \neq \emptyset$. Hence, $R S S(R) \neq \emptyset$. 